\title{
Entorno Infocomunicativo para Poblaciones Infanto- Juveniles: Activismo Digital de la Juventud Cubana Durante la Pandemia en la Habana 2020-2021
}

\author{
Info-communicative Environment for Infant-youth Populations: Digital Activism \\ of Cuban Youth During the Pandemic in Havana 2020-2021
}

\author{
Karoline Astorga González ${ }^{1^{*}}$ \\ 1 Facultad de Psicología, Universidad de La Habana, Cuba. (D) https://orcid.org/0000-0002-5880-3573 \\ * Correspondencia: karastglez951223@gmail.com
}

Recibido: 30 abril 2021 | Aceptado: 30 julio 2021 | Publicado: 13 agosto 2021

WWW.REVISTACARIBENADEPSICOLOGIA.COM

\section{Citar como:}

Astorga González, K. (2021). Entorno infocomunicativo para poblaciones infanto-juveniles: Activismo digital de la juventud cubana durante la pandemia en la Habana 2020-2021. Revista Caribeña de Psicología, 5, e5565.

https://doi.org/10.37226/rcp.v5i1.5565

\section{RESUMEN}

La pandemia por la COVID-19 ha afectado de manera crítica la vida cotidiana, dentro y fuera de Cuba. Para la Isla, esto ha venido acompañado de un agravamiento de las condiciones socioeconómicas generales por el recrudecimiento del embargo impuesto por los E.E.U.U., el detrimento del sector turístico y las exportaciones, la carencia de divisas, agudización del desabastecimiento que irremediablemente conduce al encarecimiento de los productos de primera necesidad que permanecen disponibles. No obstante, la vida en La Mayor de las Antillas ha sido sometida a modificaciones facilitadas en gran medida, por las nuevas tecnologías. Así como al mundo virtual se le asocian buenas prácticas como el activismo, también se le atañen otras que atentan contra el bienestar de los usuarios: ciberacoso, adicción a internet, etc. El objetivo que se persigue por medio de la socialización de estas nuevas prácticas para la sociedad cubana, es visibilizar la adaptabilidad de las culturas juveniles e identificar las potencialidades y amenazas del entorno infocomunicativo en este contexto.

Palabras Claves: adicción; activismo digital; ciberacoso; cibercultura juvenil

\section{ABSTRACT}

The COVID-19 pandemic has critically affected daily life, inside and outside Cuba. For the island, this has been accompanied by an aggravation of general socio-economic conditions by the upsizing of the embargo imposed by the U.S., the detriment of the tourism sector and exports, the lack of foreign exchange, exacerbation of the shortage that irretrievably leads to the rising prices of the basic-necessities items that remain available. However, life in The Greatest of the Antilles has been dramatically modified by new technologies. Just as the virtual world is associated with good practices such as activism, it is also concerned with others that violate the well-being of users: cyberbullying, internet addiction, etc. The objective pursued through the socialization of these new practices for Cuban 
society is to make visible the adaptability of youth cultures and identify the potentials and threats of the infocommunicative environment in this context.

Keywords: addiction; digital activism; cyberbullying; youth cyberculture

\section{INTRODUCCIÓN}

Muchas oportunidades de superación profesional han sobrevivido a los embates de la pandemia. Los contenidos a tratar en este documento así lo evidencian, pues se elaboró como respuesta a un módulo de curso de postgrado que ha sido convocado por el Centro de Estudios Sobre la Juventud (CESJ) y llevado a cabo de manera virtual: la IIX Edición del Diplomado en Infancias, Adolescencias y Juventudes. En dicho módulo, cuyo título fue "Infantes, adolescentes y jóvenes: nuevas alternativas para la comunicación: Internet, redes sociales y tecnologías", se instó a desplegar una serie de concepciones teóricas y análisis de acciones sociales centradas en la juventud cubana -hoy devenida cibercultura- y su vínculo con las Tecnologías de la Información y la Comunicación (TIC).

\section{CIBERCULTURAS JUVENILES}

Según citaron Cubillas y Lukianenko (2020), entre enero de 2019 y enero de 2020, en Cuba se reportaron 630 mil nuevos internauta, para un crecimiento aproximado del 8\% (Hootsuite/We Are Social, 2020). La accesibilidad a internet y la preferencia por el producto audiovisual refuerzan la identidad, el posicionamiento y empoderamiento de las culturas juveniles (adolescentes y jóvenes) y conectan con la proyección futura por medio de la integración a la propia identidad juvenil y a los procesos socioculturales que le atribuyen sentido (Hine, 2004; Urresti, 2008; Balestrini, 2010; Igarza, 2010; Artopoulus \& Urresti, 2011; Reig \& Vílchez, 2013; en Abril \& León, 2015). El usuario es agente de cambio (Pérez Pastor, 2009) y esto se comprende en la propia conversión de la audiencia en "usuarias, productoras y emisoras", entidades creadoras de sus propios referentes, no sólo como recreadoras simbólicas de significados o interpretaciones de los referentes producidos y emitidos por otros (Rosas Mantecón, 2010; en Abril et al., 2015).

La transformación generada en las prácticas culturales ha colocado a las ciberculturas juveniles como protagonistas de sus nuevas dinámicas (Urresti, 2008;
Feixa, 2011; en Abril et.al., 2015). Urresti las definió como la "representación de ámbitos de encuentro virtual entre jóvenes que, a pesar de estar distantes en el espacio, intercambian información y datos sobre cuestiones de su interés, se relacionan entre sí con encuentros reales y posteriores, se comunican a diario a través del chat y los foros $\mathrm{y}$, en ese conjunto de flujos diversos, forjan una imagen de sí mismos, de los grupos a los que pertenecen, tomando conciencia de la generación en la que se incluyen y el mundo que les rodea". Por tanto, la accesibilidad a internet representa movilidad, confort y provoca el surgimiento de nuevos escenarios económicos, sociales (Cubillas et.al., 2020), culturales y hasta políticos.

Para Hine (2004), Winocur (2009) y Cruces (2012) (citados por Cubillas et al., 2020), las desigualdades en el uso de internet no impiden que haya ganado importancia crucial en la vida diaria de adolescentes y jóvenes ni reemplazan el tiempo que estos emplean en la realización de las otras actividades en que se implican; aunque sí las reorganizan. El uso que dan los adolescentes a internet es principalmente instrumental (Sábada, 2012; en Cubillas \& Lukianenko, 2020) y las motivaciones fundamentales son la gratificación de necesidades inmediatas como el ocio, la interacción, almacenamiento y transmisión de información en nuevo soporte, estar al tanto de situaciones escolares (Trinquete, 2019), el contacto con el mercado de productos culturales; otras de orden superior como la autoconfirmación identitaria ante el/los grupo(s) de pertenencia, la visibilidad ante los otros y la supuesta seguridad que estos asocian a las conductas y discursos que protagonizan "desde la barrera" (en referencia a la pantalla como escudo) (Rubio, 2010; en Echeburúa \& De la Paz, 2010).

Entre los saldos positivos de la red y la implicación de los jóvenes en la cultura audiovisual (Roig, 2011; en Abril et al., 2015), pueden citarse la facilitación para organizarse en grupos e intercambiar materiales de la cultura mediática, para generar procesos y espacios virtuales de sociabilidad y adaptarse a los continuos avances tecnológicos por medio de la 
adquisición de conocimientos, habilidades y competencias en dicho proceso. También reorganizan y facilitan las actividades cotidianas que no tienen desarrollo específico en el espacio virtual, como el estudio o el trabajo (Hine, 2004; Winocur, 2009; Cruces, 2012; citados por Cubillas et al., 2020).

Los jóvenes han conciliado en el espacio virtual, una serie de expectativas en torno a la apertura de mercados, desarrollo del capital social, inserción laboral, nuevos vínculos interpersonales y formas de interacción, el establecimiento de relaciones románticas y sexuales, integración en grupos, o actividades propias del estudio o ejercicio profesional (Rubios, 2010). La autora reconoce la serie de modificaciones en la distribución espacial y de las relaciones que suponen una democracia participativa y actitud política de participación en la red (bajo la responsabilidad de los usuarios), a la vez que confiere a los jóvenes poder, autonomía y posibilidad de regularse y modular su tiempo; pero también señala que el hecho de que se trate de una interface no presencial, sigue existiendo en términos reales y así, cada fenómeno derivado de ella.

\section{ACTIVISMO DIGITAL}

Desde marzo de 2020, Cuba y sus ciudadanos están inmersos en la situación pandémica por causa del nuevo coronavirus SARS-Cov-2 y la enfermedad que produce, la COVID-19. En este período ha sido casi imprescindible el uso de las TIC como mecanismo para compensar el aislamiento social y la permanencia en los hogares durante las cuarentenas, mientras facilitan el reacercamiento virtual, la estabilidad comunicativa con familiares, amigos, pareja $\mathrm{u}$ otros sujetos significativos en las esferas de desarrollo de las personas; el teletrabajo como modalidad de trabajo a distancia y la actualización del acontecer nacional e internacional sobre tópicos variados.

La distancia física, las limitaciones en el trasporte público, el desabastecimiento de las "tiendas en CUP" y las interminables filas (colas) en las "tiendas en MLC", acrecentaron la mensajería por Messenger y Whatsapp como vías para coordinar y concretar la compra-venta y reventa de bienes de primera necesidad. Esto es, el comercio electrónico (Alonso, 2020), también denominado mercado virtual. El comercio digital cuenta con vías formales e informales. Los sitios habilitados por la red de tiendas del país que incluyen envío a domicilio (TuEnvío.cu, sitios de centros comerciales como Carlos III) pertenecen al primer grupo, mientras que las páginas y grupos de venta en las redes sociales (Facebook, WhatsApp, Instagram y otras aplicaciones), así como los sitios de anuncios clasificados Revolico, Porlalivre, Ofertas.cu y demás, son de administración no estatal. Esta modalidad de comercialización requiere pago por transferencia electrónica, lo que a su vez significa que la población se ha trasladado hacia las plataformas Banca Móvil y Remota, Transfermóvil y Enzona, que sirven asimismo para pagar servicios estatales. El pago de productos por tarjetas magnéticas y transferencias electrónicas es admitido en establecimientos estatales como Coopelia y algunas bodegas y tiendas que cuenten con la disponibilidad del sistema POS (máquina de punto de venta, o en sus siglas en inglés, point of sale).

Los negocios por cuenta propia, así como los del sector estatal, han redireccionado sus productos y servicios, a la vez que redimensionado el público al que dirigían sus ofertas. La causa principal: afectaciones en el sector del turismo, específicamente de tipo extranjero por las limitaciones migratorias y de vuelos, ante lo que, de forma sabia, los cuentapropistas y las instituciones han sabido aprovechar y explotar las crecientes comunidades virtuales integradas por miembros del territorio nacional; sin que ello implique la renuncia a la comercialización con el exterior y las exportaciones en la medida de las posibilidades garantizadas en estas nuevas condiciones. A este respecto, pueden citarse las ofertas de los grupos empresariales hoteleros del Ministerio de Turismo de Cuba (MINTUR): Cubanacán, Gran Caribe e Islazul; los arrendadores de viviendas en zonas urbanas, de playa y rurales; la agudización del servicio de entregas a domicilio (como el proyecto Mandao) que preserva y facilita la gestión y funcionamiento de otros negocios privados del área de la gastronomía y la compra de alimentos (los conocidos combos de comida, bebida, aseo, pagados desde el extranjero); los proyectos identitarios Beyond Roots, Q'Negra y muchos más. Los emprendedores que hacen uso de las redes sociales para visibilizar sus negocios, han introducido muy pertinentemente los sorteos o rifas, en las que integran promoción y reforzamientos positivos (recompensas materializadas en productos, dinero, servicios) a la comunidad de seguidores, instrumen- 
tada en un conjunto de reglas y requerimientos que imponen para que un usuario sea admitido como participante de dicho sorteo.

El activismo digital de los jóvenes también se ha diversificado a manera de propaganda e informaciones movilizativas o convocatorias de mayor alcance e impacto social, con motivos que reflejan los valores distintivos de la identidad nacional: ayuda humanitaria a poblaciones más vulnerables (personas de la tercera edad, con alguna discapacidad, en aislamiento domiciliario), creación de grupos online de apoyo y orientación psicológica; la participación voluntaria de jóvenes (estudiantes y trabajadores) no pertenecientes al sector de la salud en los centros de aislamiento como apoyo al personal sanitario en labores operativas (limpieza, recepción y distribución de alimentos...) y las unidades gastronómicas del Sistema de Atención a la Familia (SAF). Estos jóvenes, al compartir su experiencia en redes sociales, invitan a la imitación de estos comportamientos en otros coetáneos. Las tecnologías han exigido repensar y a la vez posibilitado la continuación de los programas de estudios de pregrado y cursos de postgrado, así como el diseño e implementación de encuestas científicas y de opinión como instrumentos de investigación. Ha sido igualmente notoria la apertura de canales y cuentas que desempeñan el "coaching", los entrenamientos físicos virtuales, los consejos nutricionales, etc.; como mismo la recreación ha contado con variedad de retos (challenges) y producción de materiales audiovisuales por profesionales o artistas aficionados.

En referencia a los vínculos de las comunidades intra y extrauniversitaria y acciones de desarrollo local y emprendimiento, se hacen notar los proyectos "B612", "Terra", o el "Citycleta", que convocan a la población a asumir actitudes más proactivas, proambientalistas y a manifestar su afición por el ciclismo, respectivamente. La descripción más elaborada de "TerrA", a voz de sus creadores, plantea que es $<<$ un proyecto diseñado por estudiantes de la Facultad de Psicología de la Universidad de La Habana, surgido en el curso 2016-2017 con el objetivo de promover estilos de vida asociados al cuidado del medio ambiente en diferentes contextos y por ende, las acciones que realiza están vinculadas con la ciencia psicológica. Desde su creación, se han llevado a cabo actividades como la recogida de materia prima, creación de cestos de basura, trabajos voluntarios y otras colaboraciones con el proyecto "Planta" de la Facultad de Biología. Actualmente crea alianzas con los integrantes de la campaña "PsicoAhorra", una atractiva y necesaria iniciativa de estudiantes de la facultad cuyo slogan "apagar las luces y encender la creatividad", convoca a desconectar equipos y conectar con las personas $>$.

Los jóvenes han desempeñado igualmente un rol significativo y notable en las redes y fuera de ellas durante el último año, en lo referente a aspectos que tocan de cerca el status quo del sistema social vigente y sus políticas. Ha sido crucial su participación (en todos los niveles y espacios, sobre todo, el digital) en campañas sociales en contra del racismo, la homofobia y otras dimensiones de discriminación (mayormente a escala global); así como eventos políticos de contenido sensible y valoraciones sociales muy controvertidas que alcanzaron alta viralidad dentro y fuera del ámbito nacional. Es el caso del Movimiento San Isidro y el 27N (jóvenes artistas que se manifestaron frente al Ministerio de Cultura) y las respuestas gubernamentales correspondientes, siendo una de ellas la Tángana en el Parque Trillo, evento en el que se personó el presidente de los Consejos de Estado y de Ministros a elección de la Asamblea Nacional del Poder Popular de la República de Cuba, Miguel DíazCanel Bermúdez. Estos ejemplos son representativos de las aspiraciones al intercambio con las instituciones, órganos estatales y han dejado como resultado producciones audiovisuales igualmente controvertidas.

\section{RIESGOS Y USO PERJUDICIAL}

El activismo digital, como demuestran los elementos anteriormente argumentados, puede tener salidas beneficiosas y desarrolladoras o de desajuste y caos, en dependencia de la legitimidad, respaldo social y consecuencias objetivas y subjetivas que deje a su paso. Los valores positivos o perjudiciales derivados del uso de las tecnologías quedan absoluta e irrebatiblemente bajo responsabilidad de los usuarios, y en el caso de ser menores de edad, padres y tutores de los mismos.

La exposición prolongada y no supervisada ante las TIC puede provocar situaciones de vulnerabilidad o trastornos psicopatológicos en niños, adolescentes y jóvenes (del lenguaje, afectivos y de socialización, intelectuales). Algunos de los peligros trasladados al 
mundo virtual son el acoso o ciberacoso, la pérdida de la intimidad por difusión de límites entre lo íntimo, lo privado y lo público o la instauración de conductas adictivas (Echeburúa \& De la Paz, 2010); la maduración precoz de niños y adolescentes por la exposición a contenido inapropiado para sus edades (Rubio, 2010); la manifestación de conductas narcisistas e histriónicas por exposición a la palestra pública y búsqueda constante de atención; retraimiento social que ocasiona analfabetismo relacional y se compensa con la construcción de relaciones sociales ficticias; afectaciones económicas como consecuencias de los altos costos de los servicios de navegación y algunos de los productos audiovisuales (videojuegos, por ejemplo); trastornos de conducta, sedentarismo y por consiguiente, la obesidad (Griffiths, 2000; Greenfield, 2009; en Echeburúa et al., 2010); estafas y otros delitos telemáticos; desinformación por la exposición, consumo acrítico y viralización de fake news (Alonso, 2010), entre otros factores que atentan contra el bienestar de las personas sin exclusividades etarias, étnicas o de otra índole. Sin embargo, más adelante se refieren factores de riesgo que aumentan dicha vulnerabilidad.

Trinquete (2019) se refirió al mundo virtual como un "escenario para el ejercicio del control, el acoso y la dominación hacia las mujeres o hacia personas con diversas orientaciones sexuales e identidades de género", en el que se reproducen comportamientos patriarcales que retroalimentan la relación de poder entre el hombre y la mujer. Son comunes las manifestaciones de violencia de género, simbólica y de dominación escudadas en la inmediatez comunicativa, el anonimato identitario y el contacto permanente con las víctimas potenciales.

El ciberacoso, como concretara Ortega (2008, 2013; en Trinquete, 2019), implica el uso de las TIC como plataforma de conductas intencionalmente hostiles y repetidas en el tiempo, que persiguen dañar la integridad de otros. Las agresiones encaminadas a ello no tienen limitantes temporales ni espaciales (como tampoco lo tienen las redes); pueden ser llevadas a cabo públicamente o en privado, tantas veces como desee el sujeto agresor, cuya identidad declara si es su voluntad o es susceptible a ser revelada por terceras personas, como también puede quedar en desconocimiento de la(s) víctima(s) por el propio anonimato. Las acciones dañinas con frecuencia comprenden las amenazas o efecto de la revelación y divulgación, sin consentimiento, de información íntima robada o cedida en confianza por la víctima (sextorsión), la creación de perfiles falsos para atacar o suplantar identidades y, en casos de serias implicaciones legales, éticas y morales, el acoso a menores de edad (grooming).

Para Echeburúa y De la Paz (2010), otro riesgo a gran escala es la posibilidad de desarrollar una adicción a internet y las tecnologías: la ciberadicción. Como a todo estado adictivo, se le asocian el síndrome de abstinencia y los síntomas inherentes a él (disforia, trastornos del sueño como el insomnio, irritabilidad psicomotriz e intranquilidad), el efecto de tolerancia y la supeditación del estilo de vida al mantenimiento de la conducta eje central de la adicción. Esto es, desinterés por temas ajenos a la conectividad, deterioro de las relaciones y desempeño en los sistemas de comunicación y actividad.

Como premisa para la inmersión futura en esta enfermedad, de acuerdo con Becoña (2006; en Echeburúa et al., 2010), está la transformación de las TIC en el instrumento de placer y el ansia por las mismas como enmascaramiento de necesidades más demandantes, ya que el mundo virtual se concibe como ámbito fértil para crear falsas identidades (relacionadas a problemas de autoestima y niveles inadecuados de autovaloración), distorsión de la percepción del mundo real circundante y aislamiento por la pérdida o detrimento del contacto social y la capacidad de autorregulación. Las necesidades que condicionan estas prácticas descontroladas pueden, por un lado, ser de alivio ante el malestar emocional que producen el aburrimiento, la soledad, emociones fuertes como la ira, etc. (Marks, 1990; Treuer, Fábian y Füredi, 2001; Potenza, 2006; citados por en Echeburúa et.al., 2010). Otras causas que fungen como factores de riesgo para desencadenar esta adicción, son los diagnósticos psiquiátricos y psicopatologías como TDAH, ciertos trastornos de ansiedad generalizada o fobias sociales; rechazo de la imagen corporal; déficit de recursos internos para la solución de problemas de la cotidianidad $\mathrm{u}$ otras circunstancias adversas, bajo nivel de resiliencia o adaptabilidad; carencias afectivas; y la intolerancia a estímulos displacenteros de tipo físico y psíquico (Yang, Choe, Balty \& Lee, 2005; Terol, Nieto, Lledó, Sánchez, Martín-Aragón, et al., 2008; Estévez, Bayón, De la Cruz \& Fernández-Liria, 2009; García del Castillo; en Echeburúa et al., 2010). 
La ciberadicción es considerada como un tipo de adicción comportamental que se distingue por la necesidad de intervención terapéutica cuyo objetivo no puede ser la extinción de las conductas de uso, puesto que el empleo de las tecnologías es imprescindible para la adaptación activa de los sujetos en la actualidad. De esta manera, el objetivo terapéutico debe centrarse entonces en el reaprendizaje del control de la conducta, planificación del tiempo libre e introducción de cambios en el estilo de vida.

Financiamiento: La presente investigación no fue financiada por alguna entidad ni patrocinador.

Conflicto de Intereses: No existen conflictos de intereses de parte de los autores de la investigación.

Aprobación de la Junta Institucional para la Protección de Seres Humano en la Investigación: No necesaria.

Proceso de Revisión: Este estudio ha sido revisado por pares externos en modalidad de doble ciego.

\section{REFERENCIAS}

Abril, J. A., \& León, G. A. (2015). Ciberculturas juveniles y consumo audiovisual: Un acercamiento a las apropiaciones de la imagen en los procesos de socialbilidad de jóvenes en el ciberespacio. Memorias del XXVII AMIC Encuentro Nacional Querétaro. Historia y aportes sociales de la investigación en México (págs. 4509-4525). Querétaro: Universidad de Sonora.

Alonso, R. (2020). El mundo digital y los medios de comunicación en tiempos de pandemia: Cuba y Cubadebate en medio de ese océano (Parte Final). Obtenido de https://www.google.com/amp/www.cubadebate.cu/especiales/2020/07/09/elmundodigital-y-lo-medios-de-comunicacion-en-tiempos-depandemia-cuba-y-cubadebate-enmedio-de-ese-oceano-partefinal/amp/

Cubillas, M. C., \& Lukianenko, S. I. (2020). Los adolescentes cubanos en la era digital. Revista Estudio, 27, 28-44.

Echeburúa, E., \& Corral, P. d. (2010). Adicción a las nuevas tecnologías y a las redes sociales en jóvenes: un nuevo reto. Adicciones, 22(2), 91-96.

Rubio, Á. (2010). Jóvenes en red, generación digital y cambio social. Monográfico Jóvenes y Nuevos Medios.

Trinquete, D. E. (2019). \#Acoso al alcance de un click: aproximación a la violencia de género en entornos digitales. La Habana.

Obra bajo licencia de Creative Commons Atribución 4.0 Internacional (CC BY 4.0).

(C) 2021 Autores. 